\title{
HIERARCHICAL AND NON-HIERARCHICAL TRIPLES
}

J. ANOSOVA, National Astronomical Observatory, Tokyo 181, Japan

L. KISELEVA, P. EGGLETON, Institute of Astronomy, Cambridge CB3 OHA, UK

AND

\section{ORLOV}

Astronomical Institute, St. Peterburgh University, St. Petersburg 198904, Russia

Triple stars and galaxies are referred to as having hierarchical or nonhierarchical structure. Thus, the dynamically stable and unstable triple systems are usually distinguished observationally by the ratio of the larger and smaller angular separation. However, (i) projection effects may hide the true configuration; and (ii) apparent hierarchical forms may occur in both types (Anosova 1986).

Numerical experiments, as well as analytical studies of dynamics of triple systems with negative total energy allowed us to formulate the general features of their evolution. One of the basic results is the following two types of dynamics of triple systems exist:

Type I: Stable systems with non-zero angular momentum and nearly periodic orbits of bodies. The character of the motion of the bodies is conserved during the evolution of the system. These systems may be separated into classes:

Class 1: True hierarchical systems of three bodies with nearly Keplerian orbits. The minimum distance between components connects the same pair of bodies during the evolution, and the hierarchical configuration of the system is always conserved.

Class 2: True non-hierarchical stable systems of three bodies. One may separate these triple systems into three subclasses:

a) Triple systems in which the motions correspond to the Eulerian or Lagrangian analytical solutions. The ratios of the distances between the 
bodies are always constant, the rotation of the system is solid, and the initial non-hierarchical configuration is conserved during the evolution.

b) Planar true non-hierarchical triple systems of the 'dumb-bell' type, in which the minimum separation between one body and the two other alternates in strict sequence, the other two bodies not approaching one another. These triple systems have a hierarchical configuration during about $10-20 \%$ of the time, and a non-hierachical one the rest of the time.

c) Three-dimensional true non-hierarchical system of three equal-mass bodies which we call a 'toroidal' system, as the region of possible motions is a torus. The minimum separation between two bodies circulates cyclicly among the three pairs. The system has a non-hierarchical configuration during about half the time.

Type II: Unstable true non-hierarchical triple system. The minimum separation between the bodies passes from one pair of bodies to another without any definite sequence. These triple systems may have hierarchical or non-hierarchical configurations.

In the case of the random initial conditions, unstable triples occur in about $90 \%$ of cases under consideration when we study the dynamical evolution of triple systems by computer simulations (Anosova 1986; Anosova $\&$ Orlov 1994). In about $10 \%$ of cases with non-zero angular momentum, hierarchical stable systems are formed. In such systems, the motions of the bodies can be described by the superposition of two perturbed Keplerian orbits.

The stability of hierarchical systems has been studied by a number of authors (see the above reviews and also Kiseleva, Eggleton \& Anosova 1994, Kiseleva, Eggleton \& Orlov, 1994 and references therein). But our simulations show that most of these criteria are not very heplful, especially when ultimately unstable systems are not yet close to disruption and appear fairly stable. We compute the dynamical evolution of hierarchical triple systems in which both orbits are initially circular, and determine the lower limit to the ratio of periods (outer/inner) for which there is dynamical stability. This ranges from 6.3 to 3.4 , as the distant body's mass (in units of the inner binary) ranges from 100 to 0.01 . We find for some mass ratios resonance-like behaviour that occurs in a limited range of initial period ratios. A variety of types of behaviour that we find when the period ratio varies near its critical value is described.

\section{References}

Anosova J. Astrophys. Space Sci., 1986, 124, 217.

Anosova, J., Orlov V., Cel.Mech.and Dyn.Astr., 1994, 59, 327.

Kiseleva, L., Eggleton P., Anosova,J. 1994. MNRAS, 267, 161.

Kiseleva, L., Eggleton P., Orlov V. 1994. MNRAS, in press 